\title{
APLICAÇÃO DO PROJECT MODEL CANVAS NO PLANEJAMENTO DE PROJETO COM ÊNFASE NO GERENCIAMENTO DE RISCOS
}

\section{Melissa Procópio de Oliveira}

Graduada em Engenharia de Produção pelo CEFET/RJ e Pós-graduada em Gestão e Gerenciamento de Projetos pela NPPG/UFRJ.

procopiomelissa@gmail.com

\section{José Luiz Fernandes}

Pós-Doutor em Engenharia (UFRJ), RJ, Brasil

Professor do Curso de Engenharia de Produção do Centro Federal de Educação Tecnológica Celso Suckow da Fonseca - CEFET/RJ.

jluizfernandes@gmail.com

\section{RESUMO}

Este artigo trata de um proposta da utilização do Project Model Canvas como suporte ao planejamento de um projeto da empresa Alfa. Ao longo do estudo, propõe-se que - Project Model Canvas seja utilizado em conjunto com a aplicação das boas práticas de gerenciamento de projetos do Project Management Body of Knowledge (PMBoK). A integração entre esses conhecimentos é detalhada através da área de riscos do projeto.

Palavras-chave: Gerenciamento de Projetos, Project Model Canvas, Análise de Riscos.

\section{PROJECT MODEL CANVAS APPLICATION IN PROJECT PLANNING WITH EMPHASIS ON RISK MANAGEMENT}

\section{ABSTRACT}

In this article is proposed to use the Project Model Canvas as support for the planning of 
an project of company Alfa. Throughout the study, is proposed that the Project Model Canvas be used in conjunction with the application of best practices in project management from Project Management Body of Knowledge (PMBOK). The integration between this knowledge is detailed through the risk area of the project.

Keywords: Project Management; Project Model Canvas, Risk Analysis.

\section{INTRODUÇÃO}

Notáveis avanços foram realizados quanto aos conhecimentos relacionados ao gerenciamento de projetos. Entre eles, destaca-se o uso do PMBoK, a relevância dada ao gerenciamento de riscos e o surgimento de ferramentais que apoiam o gerenciamento do projeto, como o Project Model Canvas. O objetivo de aplicar a integração entre as boas práticas do PMBoK e o uso do Project Model Canvas com ênfase no gerenciamento de riscos será desenvolvida por meio de um estudo de caso com o projeto denominado XPTO da empresa Alfa. 


\section{DESENVOLVIMENTO}

Entre os diversos recursos disponíveis que servem de suporte aos mais variados tipos de projeto, destaca-se o uso do PMBoK. Contudo, cada vez mais têm surgido novas ferramentas e metodologias para o gerenciamento de projetos. Considerando este fato, neste trabalho será apresentado um estudo de caso do gerenciamento de um projeto XPTO de instalação de cabos de fibra óptica para a empresa Alfa, com a realização da integração entre o PMBOK e o Project Model Canvas.

\subsection{Revisão da Literatura}

\subsubsection{Aspectos Principais do PMBoK}

O Project Management Body of Knowledge (PMBoK) é um guia que contém as melhores práticas de gerenciamento de projetos compilados pelo PMI (Project Management Institute). O PMBoK trata de uma bibliografia de referência em gerenciamento de projetos, pois identifica e descreve conceitos e boas práticas de gerenciamento, padronizando a terminologia e os processos utilizados. A 6a edição do Guia foi publicada em 2017 e contém 49 processos agrupados em dez áreas de conhecimento e em cinco grupos de processos.

Segundo o PMI (2017), uma área de conhecimento representa um conjunto completo de conceitos, termos e atividades que compõem um campo profissional, um campo de gerenciamento de projetos, ou uma área de especialização. As áreas de conhecimento e os conhecimentos são Integração, Escopo, Cronograma, Custos, Qualidade, Recursos, Comunicações, Riscos, Aquisições e Partes Interessadas.

Segundo o PMI (2017), a aplicação dos conhecimentos dessas áreas deve ocorrer de forma apropriada, considerando as especificidades de cada projeto. Assim, uma área pode ser mais relevante e mais utilizada que outra em algumas situações como, por exemplo, neste estudo no qual será evidenciada a relevância do gerenciamento dos riscos do projeto XPTO. 


\subsubsection{Aspectos Principais do PM Canvas}

O Project Model Canvas (PM Canvas) foi desenvolvido em 2013 por José Finocchio Júnior inspirado no Business Model Canvas (criado por Alexander Osterwalde em 2010).

Segundo Finocchio Júnior (2013), o Project Model Canvas pode ser usado das seguintes formas: como documento único e consistente do planejamento do projeto imediatamente seguido pela execução ou, como ferramenta preliminar para expor a lógica do projeto, servindo de base para o plano de projeto.

De acordo com Finocchio Júnior (2013), o Project Model Canvas permite montar um modelo mental do projeto através dos treze campos expostos na figura 1. Assim é possível oferecer de forma visual o entendimento do projeto.

Figura 1 -Project Model Canvas

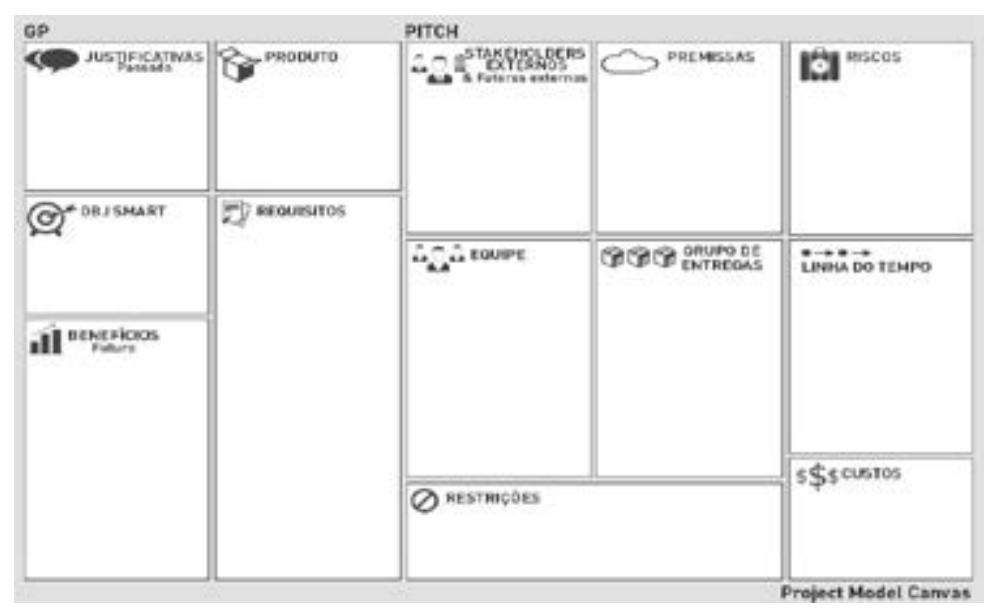

Fonte: (Finocchio Júnior, 2013)

O PM Canvas desenvolvido por Finocchio Júnior (2013) aborda os seguintes aspectos do projeto:

a) Justificativas: Apresenta as demandas não atendidas e as oportunidades não exploradas. 
b) Objetivos: Especifica o que se pretende alcançar através do projeto.

c) Benefícios: Expõe os valores tangíveis e intangíveis que serão obtidos pela organização.

d) Produto: Descreve aquilo que será entregue com características claras e mensuráveis.

e) Requisitos: Expressa o que parece necessário ou desejável no produto sob a perspectiva do cliente.

f) Stakeholders: Identifica as pessoas ou organizações envolvidas ou afetadas pelo projeto.

g) Equipe: Lista todos os que produzem algo no projeto, com seus respectivos papéis identificados no âmbito do projeto.

h) Premissas: Estabelece suposições sobre aspectos que podem influenciar o projeto.

i) Grupo de entregas: Define os componentes que fazem parte do resultado do projeto. $x)$

j) Restrições: Identifica as limitações, de qualquer origem, impostas ao projeto.

k) Riscos: Apresenta os fatores que são incertezas para o projeto.

I) Linha do tempo: Expõe as perspectivas do projeto em relação ao tempo.

m) Custos: Identifica os custos por entrega ou grupos de entrega.

\subsection{Metodologia}

O presente estudo pode ser classificado segundo Gil (2007) como exploratório quanto ao objetivo e qualitativo quanto à abordagem. Portanto, o levantamento bibliográfico e o uso de um estudo de caso elaborado são itens pertinentes, que são usados nesse estudo. 
O estudo de caso é baseado no projeto denominado XPTO pertencente à empresa Alfa que visa instalar $500 \mathrm{~km}$ de fibra óptica em valas ao lado de uma rodovia para obter uma rede de comunicação eficiente entre duas cidades. A empresa não permitiu o acesso e a reprodução integral dos documentos do projeto, no entanto autorizou o relato de algumas variáveis do projeto.

Após a apresentação dos principais aspectos acerca do PMBoK e do PM Canvas, têm-se a oportunidade de utilizar esses conhecimentos de modo conjunto no projeto XPTO. Dessa forma, será proposto um PM Canvas para o projeto XPTO e, posteriormente, será exposto como essa ferramenta pode auxiliar no gerenciamento de riscos, utilizando os conhecimentos do PMBoK.

\subsection{Estudo de caso: Projeto XPTO}

As redes de telecomunicações são de suma importância para a Empresa Alfa se comunicar com todos os seus equipamentos de instrumentação industrial de campo. Sendo assim, o projeto XPTO foi idealizado a fim de aumentar a qualidade da comunicação pois, através do uso de fibra óptica, é possível transmitir mais informações do que por via do sistema tradicional que utiliza cabos de cobre. As principais informações obtidas com a empresa Alfa sobre o empreendimento foram ilustradas no quadro 1 :

Quadro 1 - Principais dados técnicos fornecidos pelo Departamento de Engenharia

\begin{tabular}{|l|l|}
\hline Item & Descrição \\
Distância entre as Cidades & $500 \mathrm{~km}$ \\
Dimensões da Vala & $25,00 \mathrm{~cm} \times 60,00 \mathrm{~cm}$ \\
Passagem dos Cabos & 10 trechos com extensão total de $500 \mathrm{~m}$ \\
Tempo disponível para o projeto & 2 anos (aproximadamente 500 dias úteis) \\
\hline
\end{tabular}

Fonte: (Os autores)

Além das questões técnicas do empreendimento, o projeto deve estar alinhado às exigências legais de alguns órgãos públicos como o DNIT, PRF e o IBAMA. A preocupação do DNIT (Departamento Nacional de Infraestrutura de Transportes) e a (PRF) Polícia Rodoviária Federal em relação ao projeto é o impacto no fluxo da rodovia, já o IBAMA 
têm o papel de avaliar o projeto quanto aos impactos no meio ambiente.

\subsubsection{Project Model Canvas do Projeto XPTO}

A orientação de Finocchio Júnior (2013) para o preenchimento do PM Canvas baseia-se na relação deste com a ferramenta $5 \mathrm{~W} 2 \mathrm{H}$ (com exceção do fator Where - onde), que possui a seguinte sequência de questões: Why? (Por que?), What? (O que?), Who? (Quem?), How (Como?), When (Quando?) e How Much (Quanto?). Essa ordem permite estabelecer relações lógicas entre as respostas e explicitar aspectos do planejamento do projeto. Portanto, será utilizada esta sequência para a elaboração do Project Model Canvas proposto para o projeto XPTO.

Os campos que correspondem ao "Por que" do projeto são a Justificativa, os Objetivos Smart e os Benefícios, e têm por objetivo responder porque o projeto deve ser realizado, ou seja, qual é a real motivação para executar o projeto. O Produto e os Requisitos do projeto expressam "O que" será o resultado do projeto. Os recursos humanos e os stakeholders registram "Quem" são as pessoas ou grupo de pessoas que estão envolvidas na execução do projeto ou serão impactadas pelo projeto. A forma "Como" o projeto será conduzido corresponde às informações referentes às premissas e requisitos. Já os questionamentos quando e quanto correspondem, respectivamente, às informações referentes aos campos linha do tempo e custos do PM Canvas.

O PM Canvas exposto na figura 1 foi preenchido para o projeto XPTO de acordo com a sequência proposta por Finocchio Junior (2013). Os fatores de cada campo serão apresentados nas seções no quadro 2 ao quadro 13.

a) Justificativa

A dificuldade de comunicação na região em que a empresa Alfa opera resulta nas justificativas para o projeto XPTO expressas no quadro 2. 
Quadro 2 - Fatores preenchidos no campo justificativa do PM Canvas

$$
\text { Justificativa }
$$

- Falta de estrutura de telecomunicações moderna e eficiente nas instalações da Empresa 'Alfa' entre as cidades A e B.

- Ineficiência e não escalabilidade da rede telecom via cabos Ethernet.

Fonte: (Os autores)

\section{b) Campo Objetivos Smart}

O campo referente ao objetivo SMART representado no quadro 3 expressa perspectivas que o projeto deve almejar durante a sua realização.

Quadro 3 - Fatores preenchidos no campo Objetivos Smart do PM Canvas

\section{Objetivos Smart}

- Concluir $100 \%$ do projeto em até no máximo 460 dias a partir de seu início.

- Manter a realização dos custos do projeto entre as faixas de $85 \%$ a $100 \%$ dos valores orçados.

- Atingir nível de satisfação do cliente do projeto entre as faixas de $70 \%$ a $100 \%$.

Fonte: (Os autores)

\section{c) Benefícios}

Os benefícios do projeto XPTO são valores tangíveis e intangíveis que serão obtidos pela empresa Alfa após a implantação do projeto conforme observado no quadro 4.

Quadro 4 - Fatores preenchidos no campo Benefícios do PM Canvas

\section{Benefícios}

- Melhora na comunicação nos negócios da empresa Alfa entre as cidades A e B.

- Impacto positivo em outros negócios e na comunidade da região.

- Infraestrutura para futuras expansões.

Fonte: (Os autores) 
d) Produto

O projeto de instalação da fibra óptica por 500 km entre duas cidades é composto pelos produtos descritos no quadro 5.

Quadro 5 - Fatores preenchidos no campo Produto do PM Canvas

\section{Produto}

- Valas com perfurações mecanizadas;

- Instalação de subdutos;

- Caixas de passagem;

- Caixas de emenda subterrânea;

- Lançamento e fundição de fibra óptica;

- Testes ponta a ponta;

- Fechamento de valas;

- Sinalização de percurso;

- Realização de operação assistida.

Fonte: (Os autores)

e) Requisitos

O quadro 6 apresenta os fatores que são necessários ou desejáveis para o produto do projeto.

Quadro 6 - Fatores preenchidos no campo Requisitos do PM Canvas 
- Devem ser instaladas fibras ópticas entre as cidades X e Y.

- Deve contar com o apoio de órgãos públicos.

- Deve haver infraestrutura adequada para a instalação das fibras ópticas.

- Deve haver 9000 metros de valas para o lançamento de 3000 metros de fibra óptica.

- Devem ser escolhidos fornecedores considerando-se o custo total, prazo de entrega e condições de pagamento.

- Devem ser testados ponta a ponta cada trecho após instalação da fibra óptica.

- Deve ser realizada a operação assistida por 30 dias após a conclusão da instalação.

Fonte: (Os autores)

\section{f) Recursos Humanos}

A empresa Alfa possui os recursos humanos expostos no quadro 7 disponíveis para o projeto XPTO:

Quadro 7 - Fatores preenchidos no campo Recursos Humanos do PM Canvas

Recursos Humanos

- Equipe PMO: 1 Gerente de Projeto e 2 Assistentes de Projeto.

- Equipe de Engenharia: 1 Engenheiro Civil, 1 Engenheiro Elétrico, 2 Auxiliares de Engenharia e 1 Assistente Administrativo.

- Equipe de Construção Civil: 1 Encarregado, 12 Ajudantes.

- Equipe de Instalação: 1 Técnico de Fibra Óptica, 1 Encarregado e 5 Ajudantes.

Fonte: (Os autores)

\section{g) Stakeholders}

No quadro 8, pode-se observar as partes interessadas expostas no campo Stakeholders do PM Canvas e os aspectos destas que podem ter influência no projeto. 
Quadro 8 - Fatores preenchidos no campo Stakeholders do PM Canvas

\begin{tabular}{|c|c|c|c|c|c|c|c|}
\hline \multicolumn{7}{|c|}{ Identificação } & \multirow{2}{*}{$\begin{array}{c}\begin{array}{c}\text { Classifi- } \\
\text { cação }\end{array} \\
\text { Interna/ } \\
\text { Externa }\end{array}$} \\
\hline $\begin{array}{l}\text { Parte inte- } \\
\text { ressada }\end{array}$ & Relacionamento & Atitudes & $\begin{array}{l}\text { Impactos } \\
\text { Positivos }\end{array}$ & Impactos Negativos & $\begin{array}{l}\text { Grau de } \\
\text { Interesse }\end{array}$ & $\begin{array}{l}\text { Grau de } \\
\text { Influência }\end{array}$ & \\
\hline Patrocinador & Gestor & Monitoramento & $\begin{array}{l}\text { Apoio políti- } \\
\text { co, verba }\end{array}$ & $\begin{array}{l}\text { Excesso de Pressão, } \\
\text { alto intervencionismo }\end{array}$ & $\begin{array}{l}\text { 5-Muito } \\
\text { Alto }\end{array}$ & $\begin{array}{l}\text { 5-Muito } \\
\text { Alta }\end{array}$ & Interna \\
\hline $\begin{array}{l}\text { Equipe do } \\
\text { Projeto }\end{array}$ & Par & Gerenciamento & Cooperação & $\begin{array}{l}\text { Disputas internas, } \\
\text { sabotagem }\end{array}$ & $\begin{array}{l}\text { 5-Muito } \\
\text { Alto }\end{array}$ & $\begin{array}{l}\text { 5-Muito } \\
\text { Alta }\end{array}$ & Interna \\
\hline Usuários & Cliente & Informação & $\begin{array}{l}\text { Apoio, pro- } \\
\text { moção }\end{array}$ & Insatisfação & 4-Alto & $\begin{array}{l}\text { 1-Muito } \\
\text { baixa }\end{array}$ & Interna \\
\hline Fornecedores & Par & Suporte & $\begin{array}{l}\text { Entregas no } \\
\text { prazo e nas } \\
\text { especificações }\end{array}$ & $\begin{array}{l}\text { Insumos fora de } \\
\text { especificação ou com } \\
\text { defeito }\end{array}$ & 3-Médio & 3-Média & Externa \\
\hline IBAMA & Par & Monitoramento & Endosso & Veto ou proibição & $\begin{array}{l}\text { 5-Muito } \\
\text { Alto }\end{array}$ & 4-Alta & Externa \\
\hline Prefeituras & Par & Monitoramento & Endosso & Veto ou proibição & 4-Alto & 4-Alta & Externa \\
\hline Comunidades & Par & Informação & Endosso & $\begin{array}{l}\text { Pressão junto a } \\
\text { agentes públicos e } \\
\text { imprensa }\end{array}$ & 3-Médio & 2-Baixa & Externa \\
\hline DNIT & Par & Monitoramento & Endosso & Veto ou proibição & 4-Alto & 3-Média & Externa \\
\hline $\begin{array}{l}\text { Polícia Ro- } \\
\text { doviária }\end{array}$ & Par & Monitoramento & Endosso & Veto ou proibição & 2-Baixo & $\begin{array}{l}\text { 1-Muito } \\
\text { baixa }\end{array}$ & Externa \\
\hline
\end{tabular}

Fonte: (Os autores)

\section{h) Premissas}

Os fatores que devem ser considerados para o projeto XPTO e tomados como premissas são os expostos no quadro 9.

Quadro 9 - Fatores preenchidos no campo Premissas do PM Canvas

\section{Premissas}

- A realização das despesas não deverá ultrapassar o Orçamento

- Todos os fornecedores deverão ser qualificados como aptos

- Não haverá imprevistos relacionados à geografia e intempéries

- Será possível acessar todos os terrenos

\section{Benefícios}


- Não haverá problemas de tributação em função de limites municipais

- O licenciamento ambiental deverá ser emitido antes do início das atividades

- Não haverá problemas com entrega dos insumos e transporte do maquinário

- Toda a equipe deverá ser treinada e ter seu domínio técnico avaliado

- Os índices de Turn-over e Absenteísmo não deverão exceder os atuais

- Os insumos deverão ter alta qualidade e serão estocados em local coberto

- As tarefas deverão ser executadas sem falhas e de acordo com o escopo

- O pessoal terceiro contratado deve seguir os Objetivos SMART estabelecidos Fonte: (Os autores)

\section{i) Entregas}

O campo Grupo de Entregas exposto no quadro 10 apresenta como o trabalho será entregue no projeto XPTO.

Quadro 10 - Fatores preenchidos no campo Entregas do PM Canvas

\section{Entregas}

- Documentos de iniciação

- Documentos de planejamento

- Projeto Básico

- Recursos e apoio disponíveis

- Instalação completa

- Documentos de encerramento

Fonte: (Os autores)

\section{j) Restrições}

A quadro 11 apresenta as restrições que devem ser consideradas pois são limitantes para a execução do projeto XPTO. 
Quadro 11 - Fatores preenchidos no campo Restrições do PM Canvas

Restrições

- Em algumas localidades as equipes deverão residir em dormitórios temporários

- A disponibilidade dos equipamentos e maquinários é de aproximadamente $70 \%$ por não serem completamente novos

- O local a ser utilizado para armazenar os equipamentos deverá ser coberto

- As fusões devem ser realizadas em locais limpos e livres de sedimentos e partículas suspensas.

Fonte: (Os autores)

k) Riscos

A proposta desse estudo consiste na aplicação dos conhecimentos reunidos no PMBoK em relação aos riscos identificados no PM Canvas. Portanto, os Riscos identificados pelo PM Canvas serão abordados em seções posteriores.

I) Custos

O quadro 12 apresenta os custos esperados considerando Martins (2012) e IFSC (2014), para esse mesmo tipo de projeto.

Quadro 12 - Fatores preenchidos no campo Custos do PM Canvas

\begin{tabular}{|l|l|l|l|}
\hline \multicolumn{1}{|c|}{ Especificação } & \multicolumn{1}{|c|}{ Quantidade } & \multicolumn{1}{|c|}{ Valor unitário (R\$) } & \multicolumn{1}{c|}{ Valor Total } \\
\hline $\begin{array}{l}\text { Abertura de valas para lançamento de } \\
\text { dutos }\end{array}$ & 5000 metros & 30,00 & $15.000 .000,00$ \\
\hline $\begin{array}{l}\text { Instalação de caixas de passagem sub- } \\
\text { terrânea }\end{array}$ & 250 unidades & 750,00 & $187.500,00$ \\
\hline Instalação de caixa de emenda óptica & 150 unidades & 406,00 & $60.900,00$ \\
\hline Lançamento de cabo óptico & 5000 metros & 5,00 & $2.500 .000,00$ \\
\hline Fusão de cabo óptico & 42 dias & 45,00 & 1.890 \\
\hline $\begin{array}{l}\text { Certificação da rede de fibras ópticas } \\
\text { instalada }\end{array}$ & 42 dias & 15,00 & 630 \\
\hline Total Estimado & & & $\mathbf{R \$ 1 7 . 7 5 0 . 9 2 0 , 0 0}$ \\
\hline
\end{tabular}

Fonte: (Os autores) 
Para atualizar esses custos para 2019, é conveniente utilizar o IGP - M (Índice Geral de Preços do Mercado), pois este é uma média ponderada de outros índices e assim reflete com mais veracidade as mudanças na economia de forma geral. Ao utilizar a calculadora do Banco Central do Brasil (2019), observa-se o valor atualizado abaixo para o empreendimento:

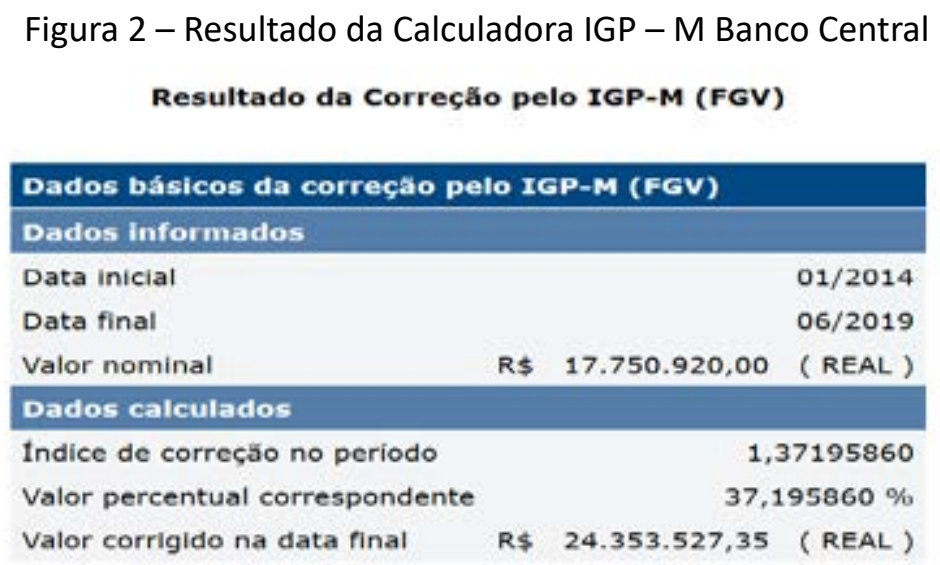

Fonte: (Banco Central do Brasil, 2019)

m) Linha do Tempo

O campo linha do tempo para o projeto XPTO considerou as durações das entregas do projeto conforme pode ser observado no quadro 13.

Quadro 13 - Fatores preenchidos no campo Linha do tempo do PM Canvas

Linha do Tempo

- Documentos de Iniciação: 7 dias

- Documentos de Planejamento: 30 dias

- Projeto Básico: 45 dias

- Recursos e apoio disponíveis: 332 dias

- Instalação completa: 291

- Documentos de Encerramento: 28 dias 
Fonte: (Os autores)

\subsubsection{Proposta de integração}

Por meio do PM Canvas elaborado para o projeto XPTO é possível compreender a complexidade do projeto que deve ser gerenciado pela empresa Alfa. No relatório Pulso da Profissão do PMI: Navegando a Complexidade, o PMI (2013) expõe que a forma que as organizações preveem, compreendem e lidam com a complexidade de seus projetos determina seus sucessos ou falhas. No mesmo relatório sobre complexidade em projetos, o PMI (2013) expõe que gerenciar mal a complexidade dos projetos acarreta em riscos tais quais o aumento do escopo, estouros de tempo e custo, falhas de comunicação e, falhas completas dos projetos.

Através do quadro 14 é perceptível que o gerenciamento de riscos do projeto é um dos principais aspectos usado sempre, ou frequentemente, no gerenciamento de projetos complexos, ocupando o primeiro lugar em organizações onde a maioria dos projetos são altamente complexos e o segundo onde poucos projetos são altamente complexos.

Quadro 14 - Uso de técnicas, métodos e práticas em projetos complexos

\begin{tabular}{|c|c|c|}
\hline \multirow{2}{*}{ Técnicas, Métodos e Práticas } & $\begin{array}{c}\text { Organizações onde a maioria } \\
\text { dos projetos são altamente } \\
\text { complexos }\end{array}$ & $\begin{array}{c}\text { Organizações onde poucos } \\
\text { projetos são altamente } \\
\text { complexos }\end{array}$ \\
\cline { 2 - 3 } & \multicolumn{2}{|c|}{ Percentual usado sempre ou com frequência } \\
\hline Prática de gerenciamento de riscos & $78 \%$ & $66 \%$ \\
\hline Medidas de desempenho de projeto & $75 \%$ & $71 \%$ \\
\hline Gerenciamento de mudanças & $74 \%$ & $62 \%$ \\
\hline Gerenciamento de recursos & $73 \%$ & $61 \%$ \\
\hline
\end{tabular}

Fonte: (Adaptado de PMI, 2013)

Através do relatório Pulso da Profissão do PMI: Captando o valor do Gerencia- 
mento De Projetos, o PMI (2015) expõe que o gerenciamento de riscos também é um importante fator para a tomada de decisões em projetos, pois se deve ponderar a gravidade e a correlação dos riscos para que se atinjam os objetivos estratégicos. Conforme pode ser observado na figura 2 , organizações que na maioria das vezes gerenciam o risco para tomar decisões têm melhores resultados quanto ao cumprimento de metas, orçamento e prazo do que em organizações que o gerenciamento de riscos é inadequado.

Figura 3 - Uso do gerenciamento de riscos para tomar decisões

Fonte: (Adaptado de PMI, 2015)

Considerando a importância do gerenciamento de riscos em projetos, é pertinente propor que para os riscos identificados no PM Canvas seja elaborada uma matriz de probabilidade versus impacto do projeto, assim como propostas de planos de ação para cada risco baseando-se nos conhecimentos contidos no PMBoK.

As probabilidades e impactos do projeto considerando, respectivamente, a frequência e a severidade, serão mensuradas na escala de 1 a 5 de acordo com suas características. Esta classificação foi baseada na probabilidade de ocorrência em projetos anteriores da empresa Alfa, tomando como base o número de eventos por unidades diárias do ano. De forma prática assumindo que em longos projetos anteriores, por exemplo, a média de incidência de um determinado risco se dá entre 2 e 3 ocorrências anualmente, assumimos então que sua probabilidade é de aproximadamente $0,5 \%$. Sendo assim pode-se definir o critério de Probabilidade conforme os quadros 15 e 16.

Quadro 15 - Classificação de Probabilidade adotada

\begin{tabular}{|l|l|l|}
\hline Probabilidade & Característica & Frequência \\
\hline $\mathbf{1}$ & Extremamente Remota & $\mathrm{p}<0,05 \%$ \\
\hline $\mathbf{2}$ & Remota & $0,05 \% \leq \mathrm{p}<0,5 \%$ \\
\hline $\mathbf{3}$ & Improvável & $0,5 \% \leq \mathrm{p}<2,5 \%$ \\
\hline $\mathbf{4}$ & Provável & $2,5 \% \leq \mathrm{p}<10 \%$ \\
\hline $\mathbf{5}$ & Extremamente Provável & $10 \% \geq \mathrm{p}$ \\
\hline
\end{tabular}


Fonte: (Os autores)

Quadro 16 - Classificação de Probabilidade adotada

\begin{tabular}{|l|l|l|}
\hline Impacto & Característica & Severidade \\
\hline $\mathbf{1}$ & Insignificante & $\begin{array}{l}\text { Degradação de atividades do projeto, gerando im- } \\
\text { pacto mínimo nos objetivos e/ou padrões. }\end{array}$ \\
\hline $\mathbf{2}$ & Baixo & $\begin{array}{l}\text { Degradação de atividades do projeto, gerando im- } \\
\text { pacto pequeno nos objetivos. }\end{array}$ \\
\hline $\mathbf{3}$ & Intermediário & $\begin{array}{l}\text { Interrupção do projeto ou de alguma de suas ativi- } \\
\text { dades, gerando impactos significativos mas rever- } \\
\text { síveis nos objetivos. }\end{array}$ \\
\hline $\mathbf{4}$ & Severo & $\begin{array}{l}\text { Interrupção do projeto, gerando impactos com re- } \\
\text { versão muito difícil nos objetivos. }\end{array}$ \\
\hline $\mathbf{5}$ & Catastrófico & $\begin{array}{l}\text { Paralisação definitiva e/ou inviabilização da con- } \\
\text { tinuidade do projeto, gerando impactos irreversí- } \\
\text { veis nos objetivos. }\end{array}$ \\
\hline
\end{tabular}

Fonte: (Os autores)

Os resultados da relação entre a probabilidade e o impacto dos riscos do projeto XPTO estão representados no quadro 17.

Quadro 17 - Probabilidade versus impacto dos riscos do projeto XPTO

\begin{tabular}{|l|l|l|l|l|}
\hline ID & Descrição do Risco & P & I & PxI \\
\hline R1 & Estouro de Orçamento & 2 & 5 & 10 \\
\hline R2 & Dificuldade de qualificação de fornecedores & 1 & 4 & 4 \\
\hline R3 & Imprevistos relacionados à geografia e intempéries & 2 & 3 & 6 \\
\hline R4 & $\begin{array}{l}\text { Impossibilidade de acesso aos terrenos, por posse da União e Mu- } \\
\text { nicípios }\end{array}$ & 1 & 3 & 3 \\
\hline R5 & Problemas de tributação entre limites municipais & 2 & 2 & 4 \\
\hline R6 & Insucesso na aquisição de licenciamento ambiental & 3 & 5 & 15 \\
\hline R7 & $\begin{array}{l}\text { Problema com entrega e logística de transporte dos insumos e ma- } \\
\text { quinário }\end{array}$ & 2 & 4 & 8 \\
\hline R8 & Falta de domínio técnico & 1 & 3 & 3 \\
\hline R9 & Altos índices de Turn-over e Absenteísmo & 1 & 3 & 3 \\
\hline R10 & Má conservação ou baixa qualidade dos insumos & 3 & 4 & 12 \\
\hline
\end{tabular}




\begin{tabular}{|l|l|l|l|l|}
\hline R11 & $\begin{array}{l}\text { Presença de divergência e/ou falhas de projeto na execução de ta- } \\
\text { refas }\end{array}$ & 1 & 4 & 4 \\
\hline R12 & $\begin{array}{l}\text { Problemas na execução em função da contratação de pessoal ter- } \\
\text { ceiro }\end{array}$ & 1 & 5 & 5 \\
\hline
\end{tabular}

Fonte: (Os autores)

De acordo com a pontuação obtida pela multiplicação entre a probabilidade e a severidade, a Matriz de Riscos do projeto XPTO ficou com a configuração demostrada na figura 3. Na Figura 4 também foi atribuído um identificador para cada risco, a ser utilizado posteriormente.

Figura 4 - Matriz probabilidade versus impacto

\begin{tabular}{|c|c|c|c|c|c|c|}
\hline Projeto XРТО & Impacto & $\begin{array}{l}\text { Insignifi- } \\
\text { cante }\end{array}$ & Baixo & $\begin{array}{l}\text { Intermediá- } \\
\text { rio }\end{array}$ & Crítico & Catastrófico \\
\hline Probabilidade & Pontuação & 1 & 2 & 3 & 4 & 5 \\
\hline $\begin{array}{l}\text { Extremamente } \\
\text { Provável }\end{array}$ & 5 & R12 & & & & \\
\hline Provável & 5 & & & & & \\
\hline Improvável & 3 & & & & R10 & R6 \\
\hline Remota & 2 & & R5 & R3 & R7 & R1 \\
\hline $\begin{array}{l}\text { Extremamente } \\
\text { Remota }\end{array}$ & 1 & & & R4 R8 R9 & R2 R11 & \\
\hline
\end{tabular}

Fonte: (Os autores)

As cores verde, amarelo e vermelho da matriz relacionam-se respectivamente com a categorização dos riscos em Não Críticos (verde - resultado entre 1 e 3), Moderados (amarelo - resultado entre 4 e 10) e Críticos (vermelho - resultado entre 11 e 25).

Os riscos Não Críticos referem-se àqueles que geram interrupção do projeto ou alguma de suas atividades, gerando impactos significativos, mas reversíveis nos objetivos. Os riscos Moderados geram a interrupção do projeto, gerando impactos com reversão muito difícil nos objetivos.

Os riscos Críticos geram a paralisação definitiva e/ou inviabilização da continui- 
dade do projeto, gerando impactos irreversíveis nos objetivos. Portanto, para cada um desses riscos devem ser tomados diferentes tipos de estratégias conforme apresentadas no quadro 18.

\section{Quadro 18 - Criticidade e estratégias a serem empregadas}

\begin{tabular}{|l|l|}
\hline Categorização & Estratégia a ser empregada \\
\hline $\begin{array}{l}\text { Não Crítico } \\
\text { (NC) }\end{array}$ & $\begin{array}{l}\text { Aceitar Passivamente: avaliar se o risco exige uma resposta, porém, sem necessi- } \\
\text { dade de alocação de recursos adicionais. }\end{array}$ \\
\hline $\begin{array}{l}\text { M o d e r a d o } \\
\text { (M) }\end{array}$ & $\begin{array}{l}\text { Aceitar Ativamente: construir um plano de ações resposta incluindo a necessida- } \\
\text { de de recursos adicionais. }\end{array}$ \\
\hline Crítico (C) & $\begin{array}{l}\text { Prevenir ou Mitigar: eliminar o fator de risco antes de executar a tarefa com a } \\
\text { qual se relaciona, ou construir um plano de ações de contingência para reduzir } \\
\text { seu impacto. }\end{array}$ \\
\hline
\end{tabular}

Fonte: (Os autores)

Por fim, para cada risco do projeto XPTO representado nos quadros 19 e 20 por seu identificador, foi elaborado um conjunto de ações a serem seguidas.

Quadro 19 - Planos de ação para mitigar os riscos

\begin{tabular}{|c|c|}
\hline ID & Ações Mapeadas \\
\hline R1 & $\begin{array}{l}\text { Realizar orçamento utilizando ao menos três métodos de precificação e decidir pelo de maior } \\
\text { valor: } \\
\text { a. Precificação histórica; } \\
\text { b. Levantamento de Custos e aplicação de margem líquida esperada; } \\
\text { c. Precificação pelo Custo de Oportunidade e Depreciação do capital. }\end{array}$ \\
\hline R2 & Buscar endosso de empresas que realizaram projetos similares. \\
\hline R3 & $\begin{array}{l}\text { Calcular os prazos com uma margem de segurança relacionada ao percentual histórico de intem- } \\
\text { péries para a região. }\end{array}$ \\
\hline R4 & Realizar mapeamento territorial para checagem prévia de posse. \\
\hline R5 & Realizar estudo tributário e definir somente uma das cidades como base de operações. \\
\hline
\end{tabular}

Fonte: (Os autores) 
Quadro 20 - Planos de ação para mitigar os riscos (Continuação)

\begin{tabular}{|c|c|}
\hline R6 & $\begin{array}{l}\text { 1. Solicitar suporte do comitê de Relações Institucionais e Corporativas da empresa durante a } \\
\text { fase de projeto. } \\
\text { 2. Solicitar apoio político dos governos estadual e federal com os quais a empresa já possui bom } \\
\text { relacionamento. }\end{array}$ \\
\hline R7 & Aumentar a frequência de visitas e pesquisas de campo. \\
\hline R8 & Aplicar testes prático-teóricos para a equipe selecionada. \\
\hline R9 & $\begin{array}{l}\text { 1. Criar metas de Remuneração Variável para bonificação; } \\
\text { 2. Aplicar a Gestão de Consequências com punições previstas pela CLT. }\end{array}$ \\
\hline R10 & $\begin{array}{l}\text { 1. Instalar bases temporárias de estocagem e distribuição dos equipamentos e insumos; } \\
\text { 2. Aprovisionar entregas mais frequentes e precisas. }\end{array}$ \\
\hline R11 & Criar um checklist de auditoria interna para o projeto. \\
\hline R12 & $\begin{array}{l}\text { Incluir no contrato cláusulas que garantam o bom nível de execução das tarefas seguindo os } \\
\text { objetivos SMART }\end{array}$ \\
\hline
\end{tabular}

Fonte: (Os autores)

\section{CONSIDERAÇÕES FINAIS}

Considerando os impactos desse projeto para os negócios da empresa Alfa, a integração exemplificada através do estudo dos riscos, seria um instrumento eficiente para simplificar a tomada de decisão, o entendimento por parte dos stakeholders e a organização das informações relevantes. No geral, o uso do PM Canvas, promove alinhamento no planejamento, criando um elo forte entre a expectativa e a realidade prática, reduzindo os desvios de execução.

Sendo assim, o estudo mostrou grande oportunidade de utilização das metodologias e ferramentas propostas aplicadas ao planejamento do projeto, organizando e dispondo as informações mais importantes de maneira mais objetiva e eficaz. Como exemplificado, o Project Model Canvas pode ser complementado através dos conhecimentos do PMBOK por ser mais estruturado com muitas técnicas e ferramentas úteis. Cabe ao gerente de projetos saber como integrar esses conhecimentos e promover o enri- 
quecimento do planejamento.

\section{REFERÊNCIAS}

BANCO Central do Brasil. Calculadora do Cidadão - Correção de Valores. Disponível em: < https://www3.bcb.gov.br/CALCIDADAO/publico/corrigirPorIndice. do?method=corrigirPocorrigi $>$. Acesso em: 20 jun. 2019.

FINOCCHIO JUNIOR, José. Project Model Canvas - Gerenciamento de Projetos sem Burocracia. 1. Ed. Rio de Janeiro: Campus, 2013.

GIL, A. C. Métodos e técnicas de pesquisa social. 4. ed. São Paulo: Atlas, 1994. Como elaborar projetos de pesquisa. 4. ed. São Paulo: Atlas, 2007.

IFSC, INSTITUTO FEDERAL DE SANTA CATARINA. Ata de registro de preços № 18/2014. Florianópolis: 2014. Disponível em: <http://www.ifsc.edu.br/arquivos/compras/AtaPE182014.pdf>. Acesso em: 20 set. 2018.

MARTIN, Paulo Caetano. Aplicação prática das metodologias tradicional e ágil no gerenciamento de um projeto. Monografia (Especialização) - Curso de Gerenciamento de Portfólio, Programas e Projetos, Escola Politécnica, Universidade Federal do Rio de Janeiro, Rio de Janeiro, 2014.

PMI, Project Management Institute. Um Guia do Conhecimento em Gerenciamento de Projetos (PMBoK) 6a ed., Rio de Janeiro, Project Management Institute, 2017. 
PMI. Pulso da Profissão do PMI: Navegando a Complexidade. Newtown Square: Project Management Institute, 2013. Disponível em: <https://www.pmi.org/-/media/pmi/documents/public/pdf/learning/thought-leadership/pulse/navigating-complexity.pdf?sc_ lang_temp=pt-PT>. Acesso em: 20 set. 2018.

PMI. Pulso da Profissão do PMI: Captando o valor do Gerenciamento De Projetos. Newtown Square: Project Management Institute, 2015. Disponível em: <https://www. pmi.org/-/media/pmi/documents/public/pdf/learning/thought-leadership/pulse/capture-value-decision-making.pdf?sc_lang_temp=pt-PT>. Acesso em: 20 set. 2018. 